\title{
Morphological and microstructural characterization of nanostructured pure $\alpha$-phase W coatings on a wide thickness range
}

\author{
N. Gordillo ${ }^{\mathrm{a}, \mathrm{b}, *}$, M. Panizo-Laiz ${ }^{\mathrm{a}}$, E. Tejado ${ }^{\mathrm{c}, \mathrm{d}}$, I. Fernandez-Martinez $^{\mathrm{e}, \mathrm{f}}$, A. Rivera $^{\mathrm{a}}$, \\ J.Y. Pastor ${ }^{\mathrm{c}}$, C. Gómez de Castrog ${ }^{\mathrm{g}}$, J. del Rio ${ }^{\mathrm{h}}$, J.M. Perlado ${ }^{\mathrm{a}}$, R. Gonzalez-Arrabal ${ }^{\mathrm{a}}$ \\ a Instituto de Fusión Nuclear, ETSI de Industriales, Universidad Politécnica de Madrid, C/José Gutierrez Abascal, 2, E-28006 Madrid, Spain \\ b CEI Campus Moncloa, UCM-UPM, Madrid, Spain \\ ${ }^{c}$ Department of Materials Science, Research Centre on Safety and Durability of Structures and Materials (CISDEM), UPM-CSIC, C/Profesor Aranguren s/ $n$, \\ E-28040 Madrid, Spain \\ d Centro Nacional de Investigaciones Metalúrgicas, CENIM-CSIC, Madrid, Spain \\ e Instituto de Energía Solar (IES), Universidad Politécnica de Madrid, Avenida Complutense s/n, E-28040 Madrid, Spain \\ ${ }^{\mathrm{f}}$ Instituto de Microelectrónica de Madrid, IMM-CNM-CSIC, Isaac Newton 8 PTM, Tres Cantos, E-28760 Madrid, Spain \\ g Departamento de Física de Materiales, Facultad de CC. Químicas, Universidad Complutense de Madrid, Ciudad Universitaria s/n, E-28040 Madrid, Spain \\ ${ }^{\text {h }}$ Departamento de Física de Materiales, Facultad de CC. Físicas, Universidad Complutense de Madrid, Ciudad Universitaria s/n, E-28040 Madrid, Spain
}

\begin{abstract}
A B S T R A C T
Nanostructured tungsten (nanoW) coatings have been deposited by DC magnetron sputtering. First, the influence of the sputtering power on the adhesion of the coatings to the substrate was investigated by depositing coatings at powers varying from 30 up to $220 \mathrm{~W}$. Non-delaminated coatings were achieved at powers $\leq 50 \mathrm{~W}$. Second, the influence of coating thickness on the morphological, microstructural and mechanical properties was investigated for films deposited at $50 \mathrm{~W}$ with thicknesses varying from $30 \mathrm{~nm}$ up to $\sim 4.0 \mu \mathrm{m}$. SEM images reveal that all the films are highly compact, consisting of nanometer sized columns that grow perpendicular to the substrate. XRD data evidence that films are monophasic, being made of pure $\alpha$ phase. All coatings show compressive stress and low micro-strain. Nanoindentation tests show that coatings have a hardness higher than that reported for coarse grained W. No significant depend-ence of the previous properties on coating thickness was observed. Finally, the influence of the substrate on coatings properties was studied, by depositing a $\mathrm{W}$ coating at a power of $50 \mathrm{~W}$ on a commercial steel substrate: no significant dependence was found.
\end{abstract}

Keywords:

Sputter deposition

Nanostructured tungsten

Micro-/macro-stresses

Nanoindentation

\section{Introduction}

Due to its properties (high melting point, low vapor pressure, low physical and chemical sputtering yields, low thermal expansion, electrical conductive properties and relative chemical inertness) tungsten- and tungsten alloy-based thin films and coatings are attractive for applications such as diffusion barriers in integrated circuits [1,2], radiation shielding [3], and kinetic energy penetrators [4]. Moreover, nowadays, W-based materials is considered the best candidate as plasma facing material in both magnetic [5-8] and laser fusion reactors [9].

W thin films have been deposited by different methods, such as electron beam, arc deposition, pulsed laser deposition, molecular

\footnotetext{
* Corresponding author at: Universidad Politécnica de Madrid, Institute of Nuclear Fusion, Jose Gutierrez Abascal 2, 28006 Madrid, Spain. Tel.: +34913363110.

E-mail address: nuri.gordillo@gmail.com (N. Gordillo).
}

beam epitaxy, high-pressure torsion [10-14], being magnetron sputtering one of the most suitable for industrial purposes. Sputtered $\mathrm{W}$ films usually present large internal stresses $[15,16]$ which can hamper their industrial applications because of film cracking and loss of adhesion to the substrate. Therefore, the control of stress is of high technological interest for further implementation of W coatings. It is worthwhile to mention that stress becomes a critical point when the film thickness increases since typically larger stresses are observed for thicker films [17]. Indeed, Maier et al. [18] reported that without specific substrate surface treatment, sufficient adhesion to the substrate was observed only for $\mathrm{W}$ coatings thinner than $1 \mu \mathrm{m}$. Different approaches have been used to reduce stress in $\mathrm{W}$ thin films by using different methods: RFsubstrate biasing [19], plasma etching of the substrate [18], using a $\mathrm{Cr}$ sticking layer before the deposition of $\mathrm{W}[20]$. However, most of these methods require double deposition procedure and plasma treatments which make somehow difficult the film deposition procedure. Recently, Karabacak et al. [21,22] have shown that in-situ 
stress reduction in $\mathrm{W}$ films is possible by using a nanostructured $\mathrm{W}$ compliant layers sandwiched between the film and the substrate. In this case, the compliant layer has been deposited by oblique angle deposition. This way of reducing stress seems to be very promising but still requires rotation of the substrate during deposition. On the other hand, as illustrated by Thornton [23], the stress state of a sputtered film can be tuned to the desired value by changing the deposition parameters (i.e. substrate temperature, working gas pressure, plasma power or chamber geometry).

Based on Thornton works, we aim to optimize the sputtered $\mathrm{W}$ coatings by tuning the sputtering parameters. In this paper, we report on the DC sputtering parameters which lead to nondelaminated, pure $\alpha-W$ phase coatings. The dependence of sample morphology, microstructure, stress/strain state and mechanical properties as a function of thickness will be discussed. In addition, a preliminary study about $\mathrm{W}$ deposition on commercial steel substrate was performed due to the interest for possible applications in the industry. In particular, coatings were deposited on steel substrate since they could be very attractive for the nuclear industry [5-9], mainly because of two reasons: (i) the fact that thick, non-delaminated coatings can be deposited at room temperature on steel without any special surface treatment and/or sacrificial layer, (ii) the self-healing behavior, which is supposed for nanostructured coatings when operating under radiation environment. In this work, the substrate influence on sample morphology and microstructure is investigated.

\section{Experimental procedure}

In order to study the plasma power influence on the adhesion to the substrate of the $\mathrm{W}$ coating, a first set of samples was deposited on (1 00 ) Si at different sputtering plasma power varying from $30 \mathrm{~W}$ up to $220 \mathrm{~W}$ while keeping constant the Ar gas pressure $\left(8 \times 10^{-3} \mathrm{mbar}\right)$ and the target-substrate distance $(8 \mathrm{~cm})$.

Once the plasma power that leads to well adhered coatings was found out, the second objective was to study the influence of coating thickness on morphology, microstructure, micro-strain/stress state and mechanical properties. For this purpose, a second set of samples with thicknesses ranging from $30 \mathrm{~nm}$ to $4.0 \mu \mathrm{m}$ was sputtered at a plasma power $\sim 50 \mathrm{~W}$, known to produce well adhered coatings and the maximum deposition rate, which is very desirable for industrial purposes.

The deposition setup consists of a high vacuum chamber (main chamber) with a base pressure in the $10^{-8}$ mbar range, a lock-in chamber, and a 2 inch diameter magnetron designed and manufactured by Nano4Energy SLNE [24]. The lock-in chamber allows loading the samples in the main chamber without destroying the high vacuum, which is very relevant for the purity of the samples. Coatings were deposited from a pure W commercial target (99.95\%) in the presence of a high pure Ar atmosphere (99.9999\%). Deposition took place at room temperature (RT). Before each growth the $\mathrm{W}$ cathode was cleaned by argon plasma etching during $5 \mathrm{~min}$ in order to avoid contamination by the possible target oxidation. It is important to note that the deposition setup was only devoted to the fabrication of $\mathrm{W}$ coatings.

For transmission electron microscope (TEM) observation a specially designed nanoW film with a thickness of about $30 \mathrm{~nm}$ was deposited onto $\mathrm{BO}_{x}$ coated $\mathrm{Si}$ substrates. The $\mathrm{BO}_{x}$ layer with a thickness of $100 \mathrm{~nm}$ was evaporated in the same experimental setup by a Joule heating filament on a (100) Si substrate. After deposition, the sample was immersed in ultra pure water to dissolve the $\mathrm{BO}_{x}$ layer and the remaining nanoW thin film was captured on a TEM grid for subsequent observation.

In order to study the possible influence of substrate material on coating morphology, a sample with a thickness of $\sim 1800 \mathrm{~nm}$ was deposited at a plasma power of $50 \mathrm{~W}$ on commercial steel substrate. Prior to deposition, the steel substrate was mechanically polished by using napless synthetic cloth. For the first rough polishing step a $0.5 \mu \mathrm{m}$ colloidal alumina was used. The second polishing was done by using a $0.03 \mu \mathrm{m}$ colloidal alumina.

Before deposition, all substrates underwent cleaning processes which consist of the following steps: (i) washing the substrate with ultra-pure water soap solution, (ii) bathing in acetone during $5 \mathrm{~min}$, (iii) immersion in an isopropanol ultrasound bath, and finally, (iv) drying by blowing with nitrogen gas.

The coating morphology and topography were characterized by optical microscope, field emission gun-scanning electron microscope (FEG-SEM) and atomic force microscope (AFM). FEG-SEM images were taken by using a JEOL JSM 6335FZEISS AURIGA microscope (Carl Zeiss, Oberkochen, Germany) operated at different acceleration voltages from 1 to $5 \mathrm{kV}$. AFM was operated in the dynamic mode (Dulcinea control system from Nanotec Electrónica S.L.). Topography data and root mean square (rms) roughness were analyzed by the free software WsxM, from Nanotec Electrónica S.L. [25].

TEM measurements were performed with a JEOL JEM 2100 microscope operated at $200 \mathrm{kV}$ and equipped with an X-ray energy dispersive spectroscopy detector.

The thickness of the samples was initially measured by profilometer (Veeco Dektak Profilometer) and further verified by cross-sectional FEG-SEM images.

Structural characterization of the samples was carried out by X-ray diffraction (XRD) using a Philips X-PERT four cycle diffractometer with a $\mathrm{Cu}_{K \alpha}(\lambda=0.15405 \mathrm{~nm})$ radiation source. $\mathrm{XRD}$ measurements were performed in Bragg-Brentano geometry.

The elemental composition and the areal density of the coatings $\left(\mathrm{at} / \mathrm{cm}^{2}\right)$ were characterized by proton induced X-ray emission (PIXE) and Rutherford backscattering spectroscopy (RBS), respectively. PIXE and RBS measurements were performed at the Institute of Ion Beam Physics and Materials Research (Helmholtz-Zentrum Dresden-Rossendorf) [26] by using $\mathrm{a} \mathrm{H}^{+}$beam at an energy of 1.2 MeV impinging perpendicular to the sample surface. The characteristic X-ray emission was collected by a Li-doped Si detector located at $120^{\circ}$ to the beam direction. Backscattered ions were detected by a standard Si-barrier detector located at an angle of $170^{\circ}$ with respect to the beam direction.

In order to evaluate the mechanical response of the coatings, nanoindentation tests were carried out by using a MTS Nanoindenter XP and the continuous stiffness measurement technique together with a diamond Berkovich indenter. Hardness and elastic modulus were obtained by an indentation depth of $10-15 \%$ of the coating thickness to avoid the influence of the substrate. These values were determined from the unloading part of the force-depth $(F-d)$ curve according to the procedure reported in the literature [27]. A minimum of fifteen indentations were done on each sample.

\section{Results and discussion}

\subsection{Optimization of the sputtering parameters to achieve non-delaminated coatings}

The influence of sputtering power on the adhesion of the coatings to the substrate was studied by varying the sputtering power from 30 to $220 \mathrm{~W}$ while keeping fixed other sputtering parameters (i.e. gas pressure, cathode-substrate distance and substrate temperature). A brief overview of the sputtering parameters, deposition rate, sample thickness and adhesion to the substrate is illustrated in Table 1.

The adhesion of the coatings to the substrate is firstly investigated by optical microscopy. In Fig. 1, the images for two 

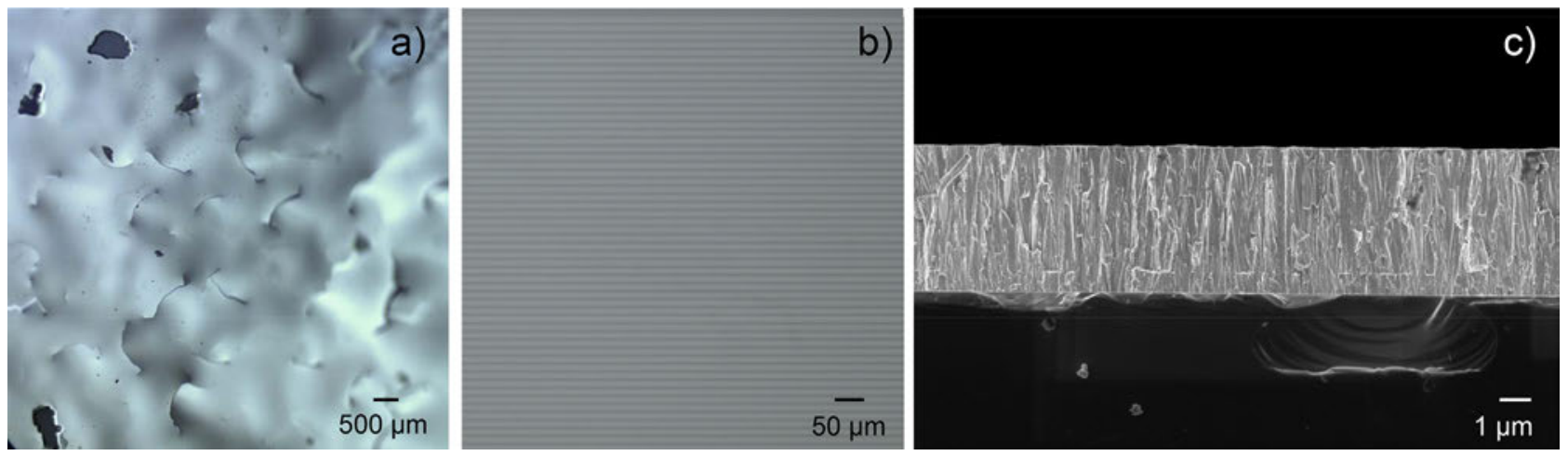

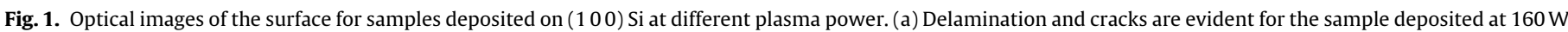

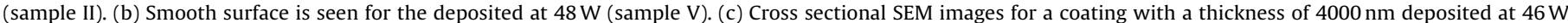
(sample VII).

representative samples (II and V) are shown. For films deposited at sputtering power larger than $50 \mathrm{~W}$, the surfaces are clearly delaminated. As an example, an optical image for a film deposited at $160 \mathrm{~W}$ is shown in Fig. 1(a). In this figure large color contrast is observed. Grey color regions correspond to parts of the sample where $\mathrm{W}$ is still present (scarcely sticking) whereas black regions correspond to naked Si surface after W has been completely peeled off. Similar delamination behavior for $\mathrm{W}$ has been previously reported to be due to the high compressive stress stored in this kind of films $[21,22]$ related mainly to the "atomic peening" process [23]. For films deposited at sputtering power $\leq 50 \mathrm{~W}$, no delamination is observed, here the optical image, Fig. 1(b), reveals homogeneous surface, which is a first indication of adhesion to the substrate. In order to have a deeper insight about the adhesion of the coating to the substrate, SEM measurements were carried out. A typical cross-sectional SEM image of a layer deposited at $46 \mathrm{~W}$ is shown in Fig. 1(c). In this figure a sharp but net interface between the W coating and the $\left(\begin{array}{lll}1 & 0 & 0\end{array}\right)$ Si substrate is seen. Thus, SEM data corroborate optical microscope images, illustrating that the interface between coatings and substrate is good. Indeed, it is worthwhile to mention that coatings sputtered at powers $\leq 50 \mathrm{~W}$ pass the scotch tape test.

According to these data, depending on the sputtering power, two regions can be identified: Region I (sputtering power $\leq 50 \mathrm{~W}$ ), samples are adhered to the substrate, and Region II (sputtering power $>50 \mathrm{~W}$ ), samples exhibit delamination.

\subsection{Thickness dependence of the morphology, microstructure and stress state of the $W$ coatings}

Once deposition parameters were optimized to achieve adhered coatings, the influence of coating thickness on the morphological

\section{Table 1}

Sample code, substrate, plasma power, thickness, deposition rate and adhesion of the samples studied in this work. The growth pressure was kept constant in all cases at $8 \times 10^{-3}$ mbar.

\begin{tabular}{llllll}
\hline $\begin{array}{l}\text { Sample } \\
\text { code }\end{array}$ & Substrate & $\begin{array}{l}\text { Plasma } \\
\text { power }(\mathrm{W})\end{array}$ & $\begin{array}{l}\text { Thickness } \\
(\mathrm{nm})\end{array}$ & $\begin{array}{l}\text { Deposition } \\
\text { rate }(\mathrm{nm} / \mathrm{min})\end{array}$ & Adhesion \\
\hline I & Si & 220 & - & - & $\times$ \\
II & Si & 160 & - & - & $\times$ \\
III & Si & 100 & - & - & $\times$ \\
IV & Si & 50 & 30 & 15 & $\sqrt{ }$ \\
V & Si & 48 & 2000 & 20 & $\sqrt{ }$ \\
VI & Si & 46 & 600 & 20 & $\sqrt{ }$ \\
VII & Si & 46 & 4000 & 17 & $\sqrt{ }$ \\
VIII & Si & 45 & 1200 & 20 & $\sqrt{ }$ \\
IX & Si & 45 & 250 & 17 & $\sqrt{ }$ \\
X & Si & 30 & 200 & 13 & 10 \\
XI & Steel & 47 & 1800 & 10 & \\
\hline
\end{tabular}

and microstructural properties as well as, on residual stress, microstrain and mechanical properties of the films was studied. For this purpose, a series of samples with thicknesses varying from $30 \mathrm{~nm}$ to $4.0 \mu \mathrm{m}$ was deposited on Si (samples from IV to IX) at fixed sputtering parameters (see Table 1 .

\subsubsection{Morphology}

FEG-SEM top view and cross-sectional images are shown in Fig. 2 for coatings with different thickness. All coatings exhibit very similar morphology constituted of columns that grow perpendicular to the substrate. Columns present an inverted pyramidal shape which is compatible with the zone $\mathrm{T}$ in the Thornton's morphology diagram [28]. Because of the pyramidal shape, the column average diameter close to the sample surface became larger with increasing sample thickness, being $\sim 70 \mathrm{~nm}$ in width for the coating with a thickness of $250 \mathrm{~nm}$ film and $\sim 400 \mathrm{~nm}$ for that with a thickness of $4.0 \mu \mathrm{m}$. Such evolution is illustrated by the histograms depicted on Fig. 2.

Fig. 3 shows AFM images of two meaningful samples ( $\mathrm{X}$ and VIII). The topography is similar to that observed by SEM images in-plane (left column on Fig. 2). The coating surface is regular and smooth. The root mean square (rms) roughness, as extracted from the quantitative height values, is lower than $3 \mathrm{~nm}$ for all samples.

One important parameter to be considered when dealing with nanostructured materials is their grain boundary architecture, since it highly influences the nanomaterial properties (i.e. radiation-resistance, mechanical properties) [29]. Because of this reason, the nature of grain boundaries in the sputtered coatings was investigated by TEM. Dark field TEM image of a $30 \mathrm{~nm}$ in thickness nanostructured W sample, especially designed for TEM observation (see experimental) are depicted in Fig. 4(a) and (b). In these figures, grains presenting anisotropic shapes, with sizes ranging from 50 to $150 \mathrm{~nm}$ are seen.

All morphological data evidence that samples microstructure is quite dense. Indeed, cross-sectional FEG-SEM images reveal the absence of voids in between columns, within the SEM resolution limit. This result differs from those reported for similar materials deposited by oblique angle deposition in which voids, associated to the shadowing effect taking place during sputtering, are typically present among the columns [30]. Then, the lack of voids may indicate that energetic ions are the main responsible for the columnar growth.

Additional information about the compactness of the coatings can be obtained from density measurements. The average coating density, estimated by combining RBS and SEM measurements, was calculated to be $19.50 \pm 0.30 \mathrm{~g} / \mathrm{cm}^{3}$. This density value is similar to 
a)

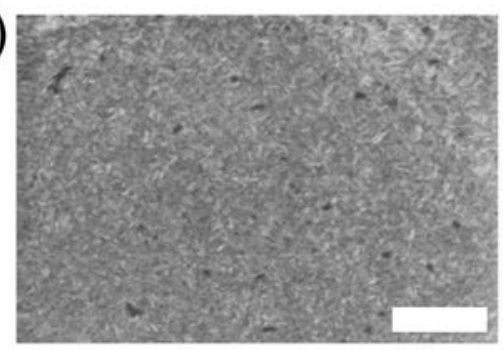

b)

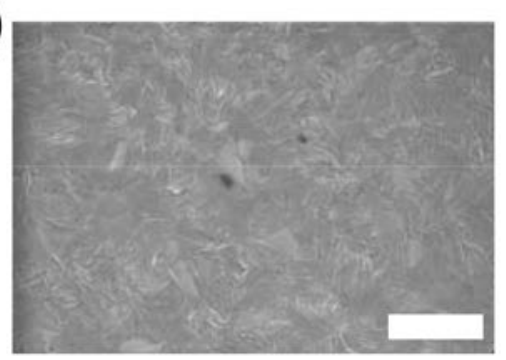

c)

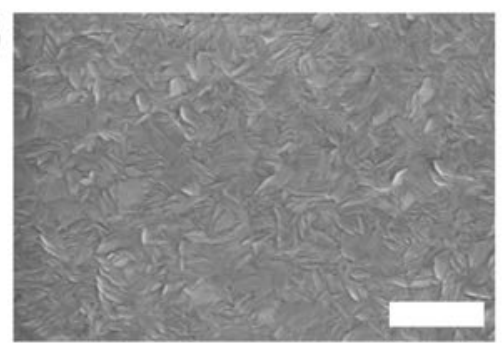

d)

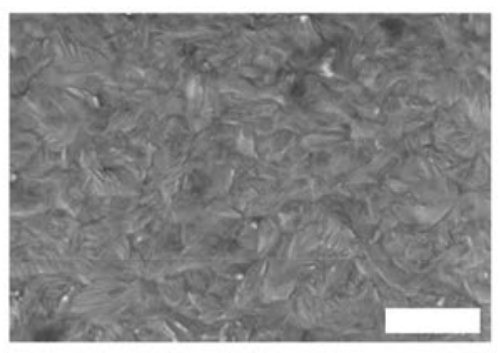

e)

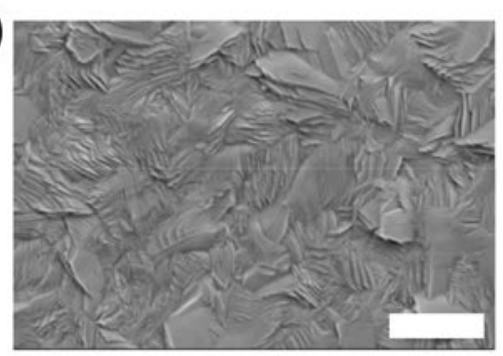

f)

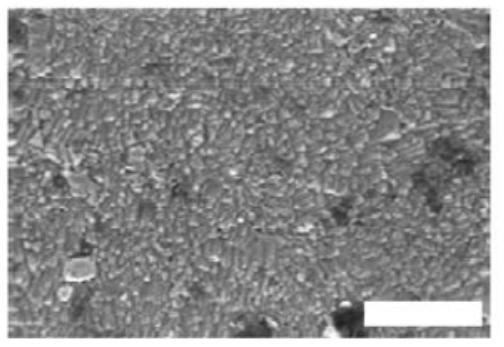

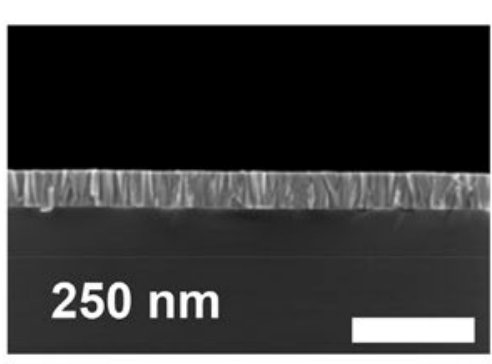
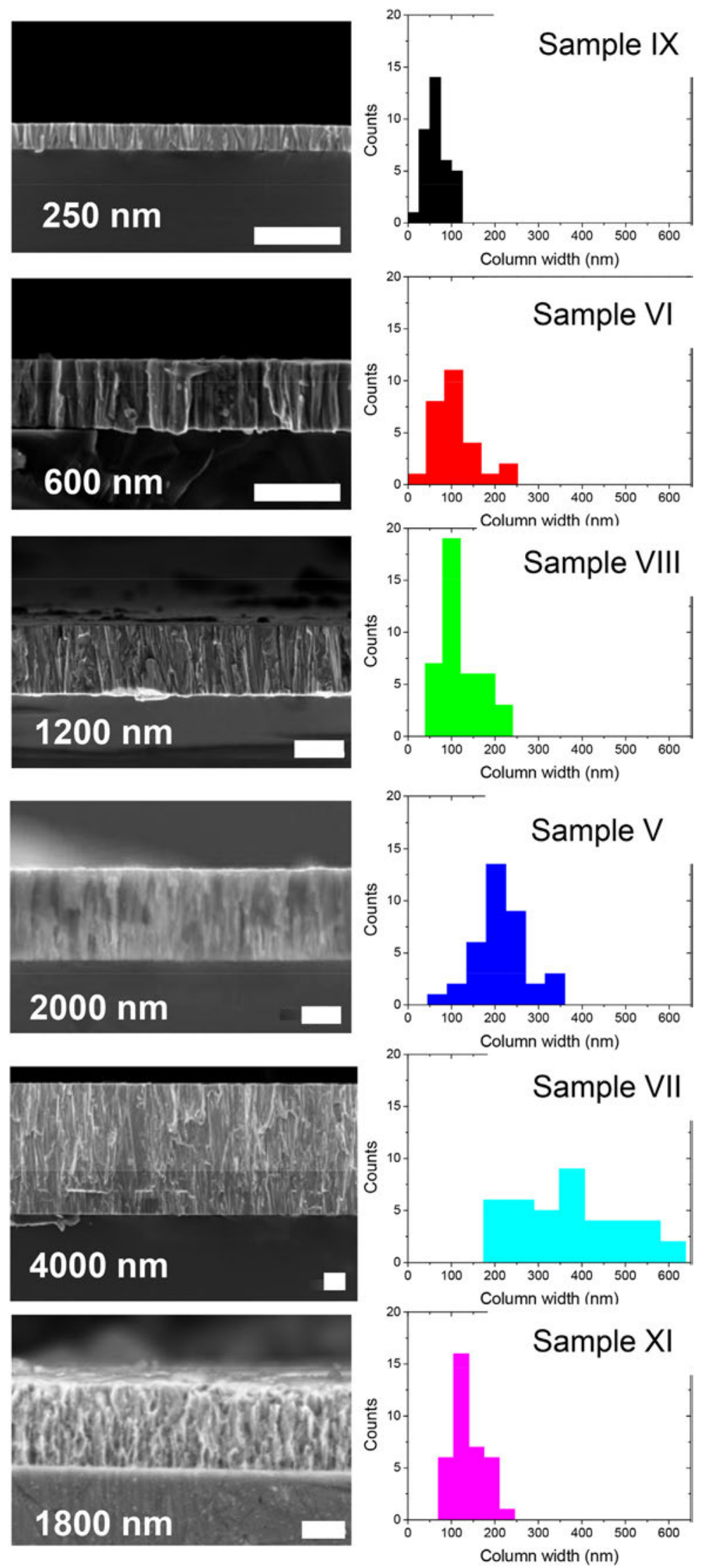

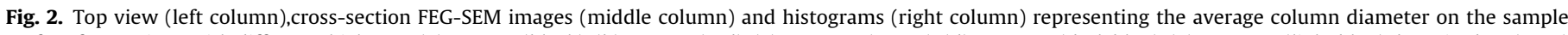

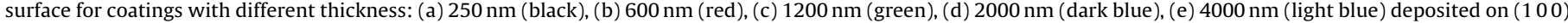
Si and (f) $1800 \mathrm{~nm}$ (magenta) deposited on commercial steel substrates. The scale bar is $800 \mathrm{~nm}$ for all photos and the thicknesses are indicated on each image. 

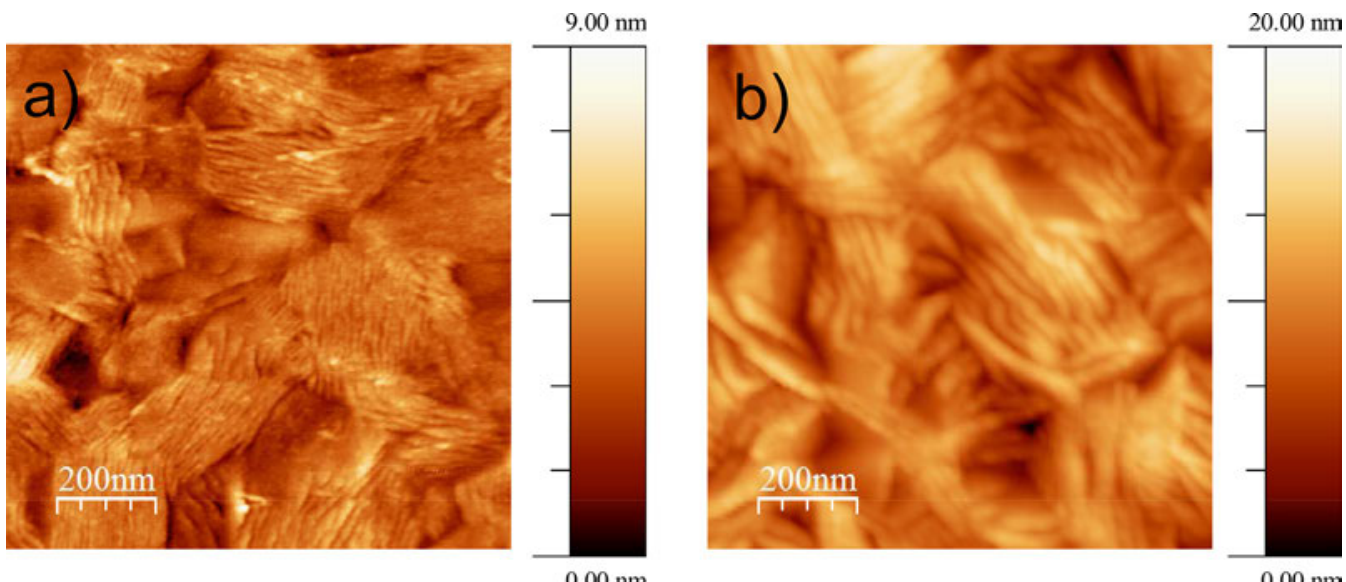

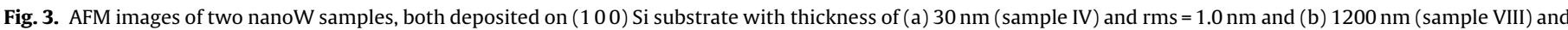
$\mathrm{rms}=2.5 \mathrm{~nm}$

that tabulated for pure coarse grained $\mathrm{W}\left(19.25 \mathrm{~g} / \mathrm{cm}^{3}\right)$ [31], which indicates that we are dealing with highly compacted coatings.

\subsubsection{Microstructure}

Fig. 4(c) shows a selected area electron diffraction (SAED) ring pattern from a region of Fig. 4(a). This ring pattern indicates that $\mathrm{W}$ film is polycrystalline. The ring pattern is well indexed by the $\alpha-\mathrm{W}$ (space group $I m-3 m$ ) structure, Fig. 4(c). Other authors report the presence of some rings assigned to both $\alpha-W$ and $\beta$ W structures [32,33] for similar as-deposited nanostructured $W$ films. They conclude that both phases of $W$ were presented in their films. Regarding the $\beta$-phase (A15), $\beta-W\{210\}$ and $\beta-W\{211\}$ are the most intense reflections. The $\beta$-phase (space group Pm$3 n$ ) is mainly seen for thin films. This phase is metastable but it may be stabilized by impurities (e.g. oxygen) [33,34]. Shen et al. [34] reported that the $\beta$-phase is stabilized when $O$ concentrations larger than $5-15 \%$ are reached.

In our case, since the interplanar distance $(d)$ of $\alpha-W\left\{\begin{array}{lll}1 & 1 & 0\end{array}\right.$ $\left(d_{\alpha-\mathrm{W}\{110\}}=0.2238 \mathrm{~nm}\right)$ and $\beta-\mathrm{W}\{210\} \quad\left(d_{\beta-\mathrm{W}\{210\}}=0.2256 \mathrm{~nm}\right)$ are similar, reflections are very close and cannot be distinguished in the ring pattern depicted in Fig. 4(c). However, if the $\beta$-phase were present in our coatings, the $\beta-W\{211\}$ reflection $\left(d_{\beta-\mathrm{W}\{211\}}=0.2062 \mathrm{~nm}\right)$ with a relative intensity (I), $\mathrm{I}_{\beta-\mathrm{W}\{211\}} / \mathrm{I}_{\beta-\mathrm{W}\{210\}}=0.96$, would be observed in the SAED pattern between $\alpha-\mathrm{W}\{110\}$ and $\alpha-\mathrm{W}\left\{\begin{array}{lll}2 & 0 & 0\end{array}\right\}$ reflections, but this is not the case. Other SAED spots measured at different regions were analyzed and the same ring pattern was obtained. The fact that we do not observe any sign of the $\beta$-phase, indicates that this phase could not be stabilized, which excludes an O concentration beyond $5-15 \%$ [34]. Apart of this indirect estimate, we could not determine the $O$ concentration, either with RBS or with PIXE because the sensitivity limit of both techniques to light species is very poor.

XRD diffraction patterns for films with different thickness are depicted in Fig. 5. All coatings exhibit four Bragg peaks corresponding to the $\alpha-\{110\}, \alpha-\{200\}, \alpha-\{211\}$ and $\alpha-\{220\}$ reflections of the thermodynamically stable body-centered cubic (bcc) $\alpha-W$ phase [17]. These results are in agreement with the SAED pattern as previously discussed, indicating that coatings are polycrystalline and monophasic (pure $\alpha$-phase). We carried out PIXE experiments to detect the presence of $\mathrm{Ar}$, a common impurity in coatings deposited by sputtering. The results (not shown) reveal that the Ar content in the coatings is $\sim 0.04 \%$, notably lower than typical reported values $(\sim 2 \%)[35,36]$.

A deeper analysis of the XRD patterns allows us to obtain more information about the film total stress and micro-strain. The film total stress was evaluated by analysing the centroid $\alpha-\mathrm{W}\{110\}$ peak profile in comparison to that reported for the conventional W, Fig. 6(a). The Si peak in Fig. 5 corresponds to the substrate and is used as an internal reference. Then, any shifts in the $2 \theta$ positions of $\mathrm{W}$ to the tabulated ones can be attributed to stress in
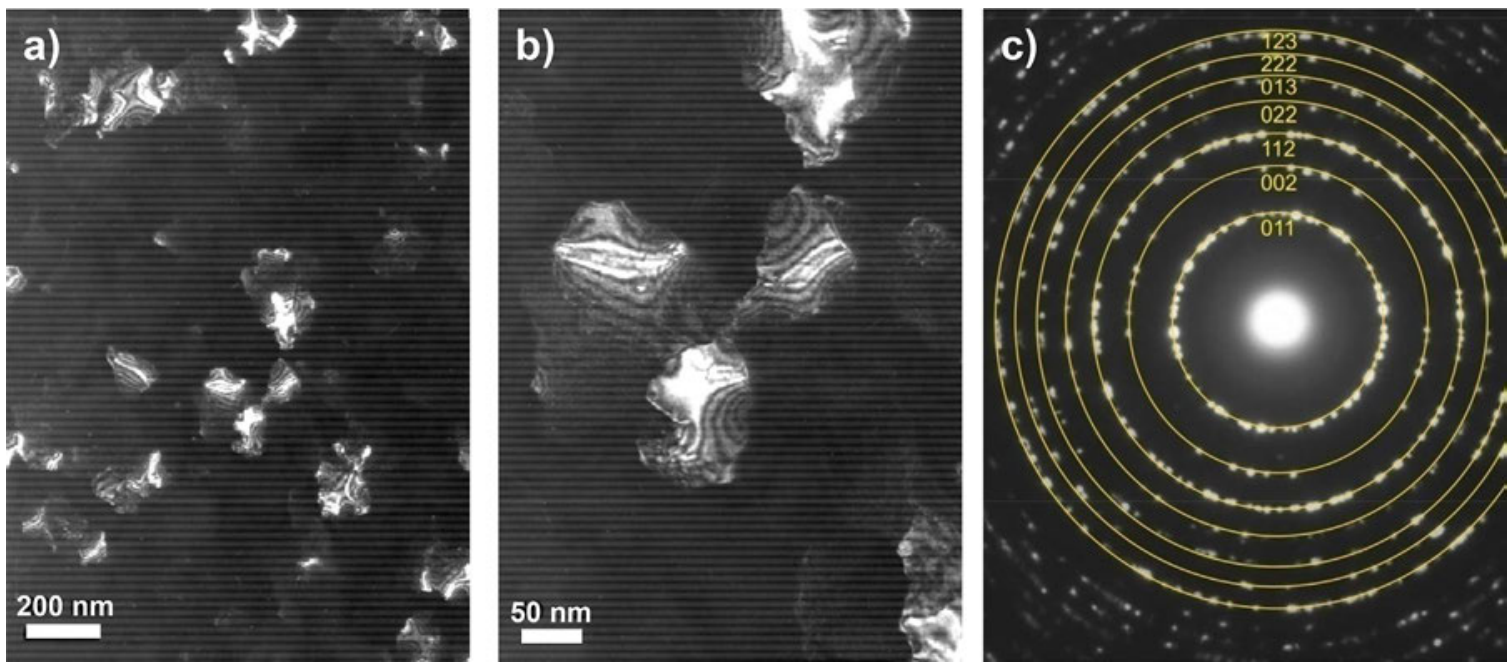

Fig. 4. Dark field TEM images measured at two different scales (a), (b) and SAED pattern (c). 


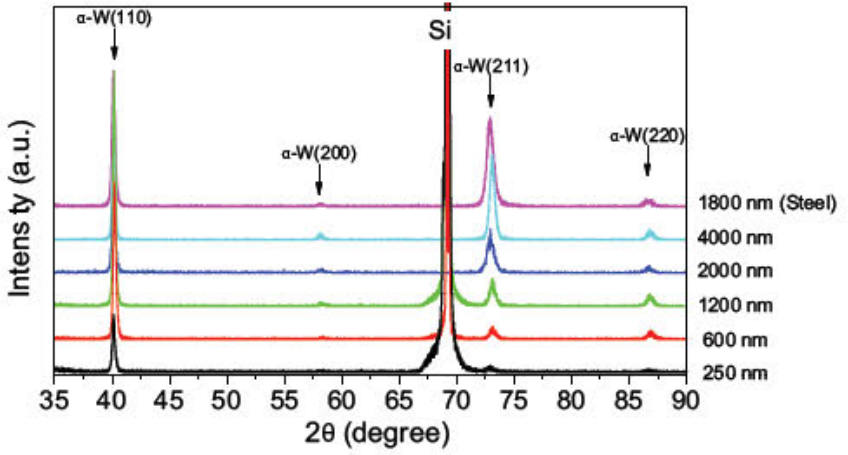

Fig. 5. X-ray diffraction patterns of nanoW coatings with different thickness: $250 \mathrm{~nm}$ (black), $600 \mathrm{~nm}$ (red), $1200 \mathrm{~nm}$ (green), $2000 \mathrm{~nm}$ (dark blue), $4000 \mathrm{~nm}$ (light blue) deposited on (100)Si substrate and nanoW sample with thickness of $1800 \mathrm{~nm}$ (magenta) deposited on a steel substrate.

the coatings. Taking this into account, the diffraction peaks corresponding to the $\{110\}$ orientation shown in Fig. 6(a), slightly shift to a $2 \theta$ position lower than that reported for conventional $\mathrm{W}$ $\left(2 \theta_{\{110\}}=40.265^{\circ}\right.$ [37]). This shift indicates the existence of larger atomic plane spacing and it originates from a compressive stress in the film. These results agree with those previously reported in literature, in which films composed predominately of the $\alpha$-phase are under compression [38]. As illustrated in Fig. 6(a), all diffraction peaks are symmetric and can be fitted by using a single Gaussian, indicating that samples have a homogeneous stress profile corroborating the presence of the pure $\alpha$-phase. The lattice parameter (a), estimated from the location of the $\{110\}$ peak, is depicted in Fig. 6(b) for films with different thickness. No significant dependence of lattice parameter on sample thickness is observed. In all cases, it is slightly larger $(\sim 0.2 \%)$ than the equilibrium one tabulated for the $\alpha-W(a=3.1648 \AA)$.

The film total stress parallel to the film surface can be evaluated according to the following expression derived from the Hooke's law:

$\sigma=-\frac{E}{v} \times \frac{\Delta d}{d}=-\frac{E}{v} \times \frac{d-d_{0}}{d_{0}}$

where $d$ and $d_{0}$ are the measured and unstrained atomic inter-planar spacing in the preferential orientation $\left.\left(\alpha-W_{\{1} 10\right\}\right)$, respectively. $E$ is the Young's modulus (410 GPa [31]) and $v$ is the Poisson's ratio $(0.28$ [31]) of the conventional W. On this basis, from the expression (1), it is possible to calculate the total residual stress in $\{110\}$ direction. The results are plotted in Fig. 6(c). The total residual stress does not depend on the coating thickness and oscillates from -2.0 up to $-5.0 \mathrm{GPa}$. These stress values agree quite well with those reported in literature for similar films with the same thickness range. For example, Kaazt et al. obtain a value about $-6.0 \mathrm{GPa}$ for samples with a thickness of $20 \mathrm{~nm}$, Ely et al. report stress values in between -4.8 and $-6.0 \mathrm{GPa}$ for samples with a thickness of around $600 \mathrm{~nm}$ and Vink et al. show a constant internal stress of $-2.1 \mathrm{GPa}$ for samples thickness between 50 and $200 \mathrm{~nm}$ $[17,39,40]$.

The coating micro-strain $(\varepsilon)$ was evaluated by the Williamson-Hall method [41] as shown in Fig. 7. The data analysis was performed from the broadening of the XRD profiles for each sample thickness by using Eq. (2):

$\beta \cos \theta=4 \varepsilon \times \sin \theta+\frac{K \lambda}{D}$

where $\beta$ is the full width at half maximum (FWHM) of the diffracted peaks, $\theta$ is the diffraction angle, $K$ is the shape factor (typically $0.9,[41])$ and $\lambda$ is the wavelength of the used radiation source and $D$ is the average grain size. Fig. 7(a) shows $\beta \cos \theta$ as a function of $\sin \theta$ and a clear linear trend is observed. Therefore, the calculated points are linearly fitted by using the mean square method. From these data, the mean average micro-strain (from the slope of the plot) as a function of thickness can be estimated, Fig. 7(b), indicating that the crystals exhibit low micro-strain. No significant dependence of the $\varepsilon$ value on sample thickness is observed.

\subsubsection{Mechanical properties}

The mechanical properties (Hardness $(H)$ and Young's modulus $(E)$ ) for coatings with different thicknesses were calculated by using the Oliver-Pharr method. The hardness and the elastic modulus of the nanostructured films, depicted in Fig. 8(a) and (b), respectively, are nearly constant within the studied thickness range. Indeed, the average hardness estimated is the same, $15.0 \pm 0.7 \mathrm{GPa}$. The hardness is significantly higher than that reported in literature for coarse-grained W (3.9 GPa) [42] and smaller than that reported for W films with a grain size of $\sim 30 \mathrm{~nm}(\sim 24 \mathrm{GPa})$ [43], which evidences the important role of the grain size in the film hardness. The average Young's modulus results to be $351 \pm 11 \mathrm{GPa}$ and is lower than Young's modulus reported for coarse-grained W (410 GPa). The results obtained from the nanoindentation test may suggest that nanostructurization induces embrittlement of the material
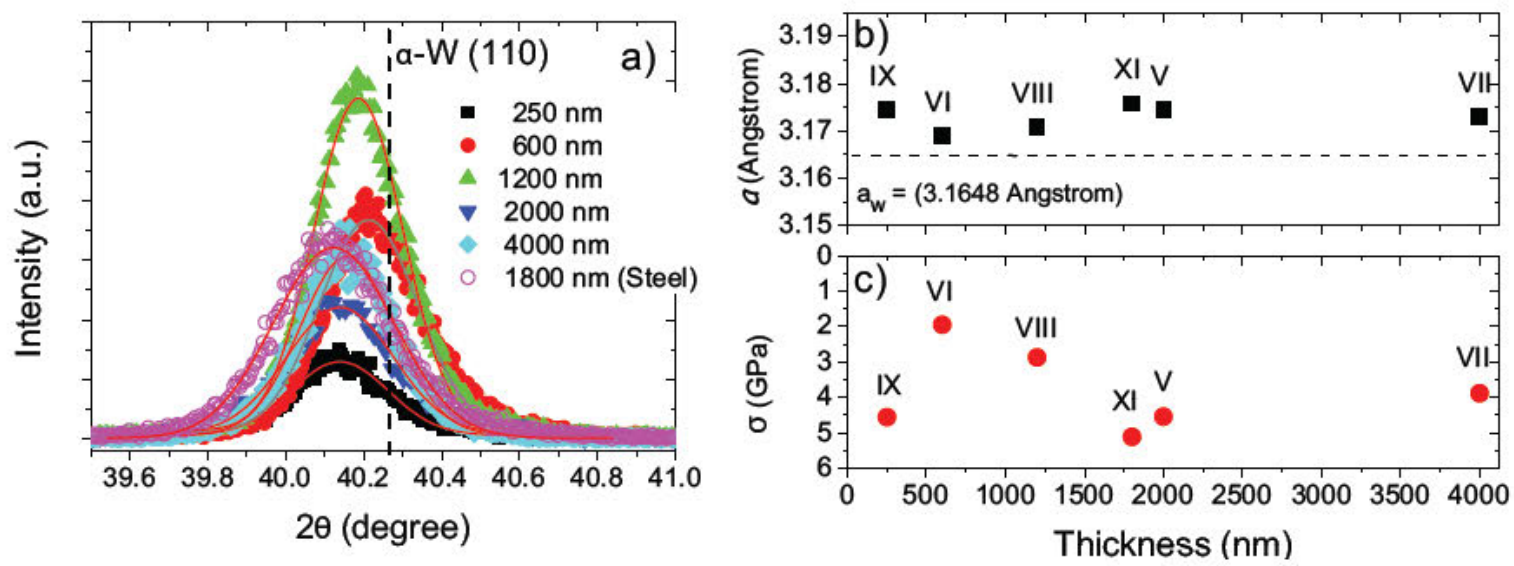

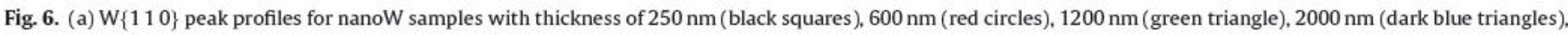

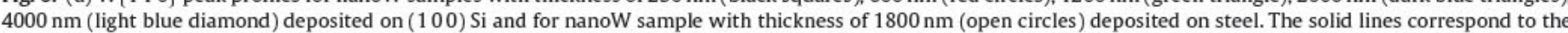

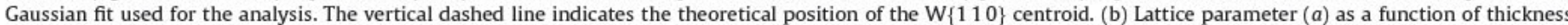

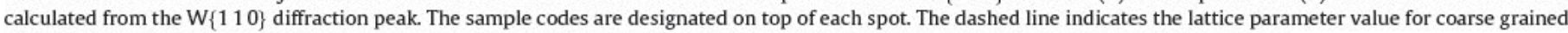
W. (c) Total residual stress obtained from the $\{110\}$ centroid as a function of thickness. The sample codes are designated on top of each spot. 

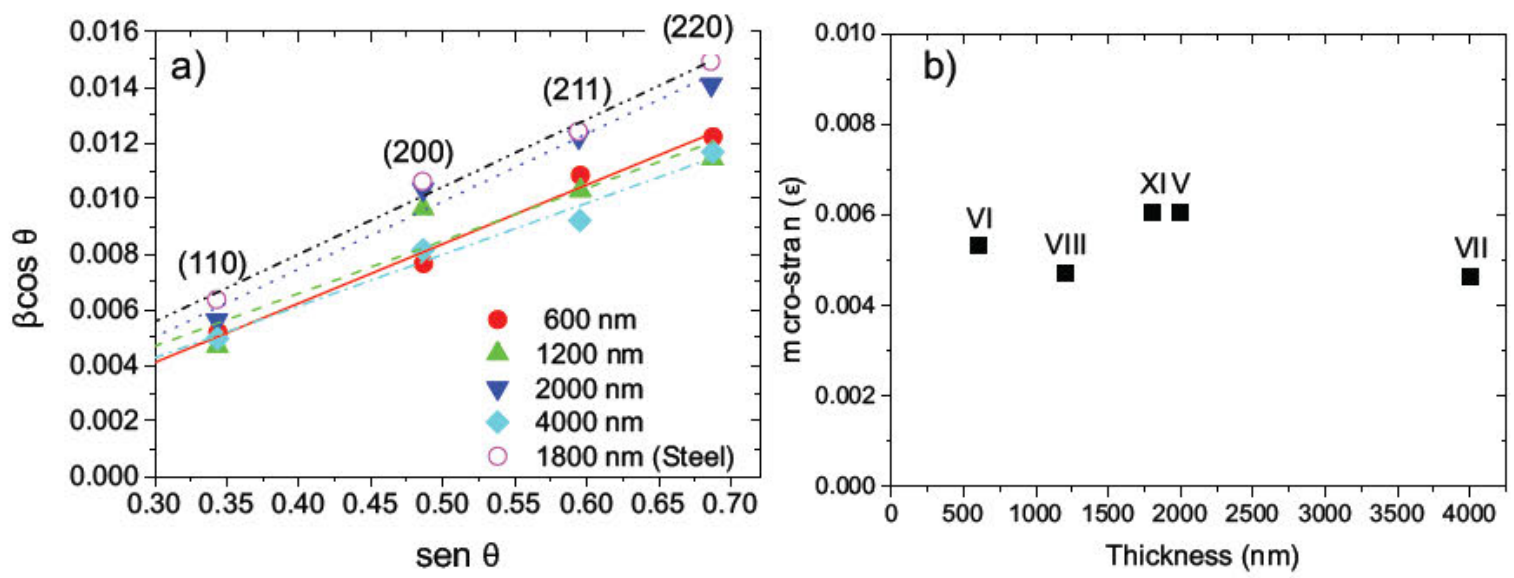

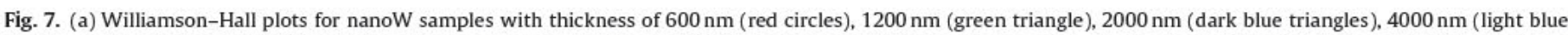

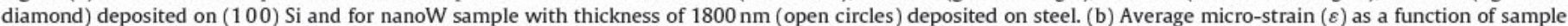
thickness. The sample codes are designated on top of each spot.

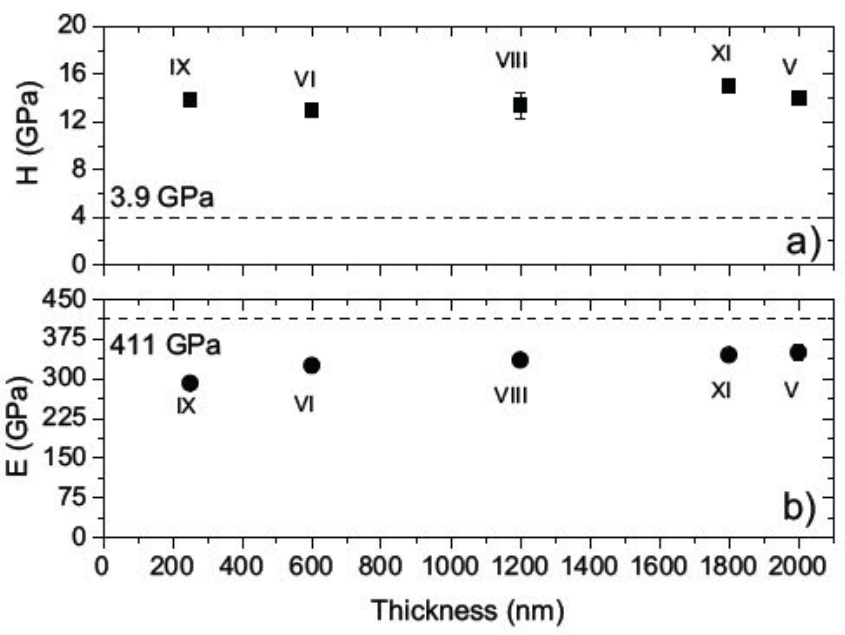

Fig. 8. (a) Hardness (black squares) and (b) Young's modulus (black circles) as a function of thickness for nanoW coatings deposited $\mathrm{Si}$ and on steel substrates. The horizontal dashed lines indicate the theoretical values of the conventional $\mathrm{W}$. The sample codes are designated on top of each symbol.

although a deeper study on the brittle-ductile transition would be needed in order to assess a better conclusion. This study is planned in further work. Fig. 9(b) shows the typical load-displacement curve for the performed nanoindentation tests for samples with similar thickness and deposited on different substrates. It is continuous during loading, without any step in the displacement axis, which is an indirect measurement of the good adhesion of the films to the substrates.

\subsection{Influence of the substrate}

The morphology of the coatings may not be only influenced by the sputtering parameters but also by the substrate properties. In order to investigate, if there exists any influence of the substrate on coating deposition, morphology, microstructure, stress and strain state, as well as, on the mechanical properties, a film with a thickness of $1800 \mathrm{~nm}$ (medium range) was deposited on a commercial steel substrate (sample XI). The deposition conditions are listed in Table 1. It is worthwhile to mention that they are similar to that used for the deposition of a coating with a similar thickness $(2000 \mathrm{~nm})$ on $(100) \mathrm{Si}$ (sample V). As shown in Fig. 2(f), the W coating deposited on steel also consists of tightly packed columns growing perpendicular to the substrate with a mean diameter at sample surface of $\sim 120 \mathrm{~nm}$. From the morphological point of view, no differences between the coating sputtered on steel and that sputtered on (100) Si are observed. The XRD pattern of the coating deposited on steel is shown in Fig. 5. According to these data, the nanoW film has also a polycrystalline structure made of a pure $\alpha-W$ phase and preferentially oriented along the $\left\{\begin{array}{ll}1 & 10\end{array}\right.$ direction. It also exhibits compressive stress with a similar lattice parameter and
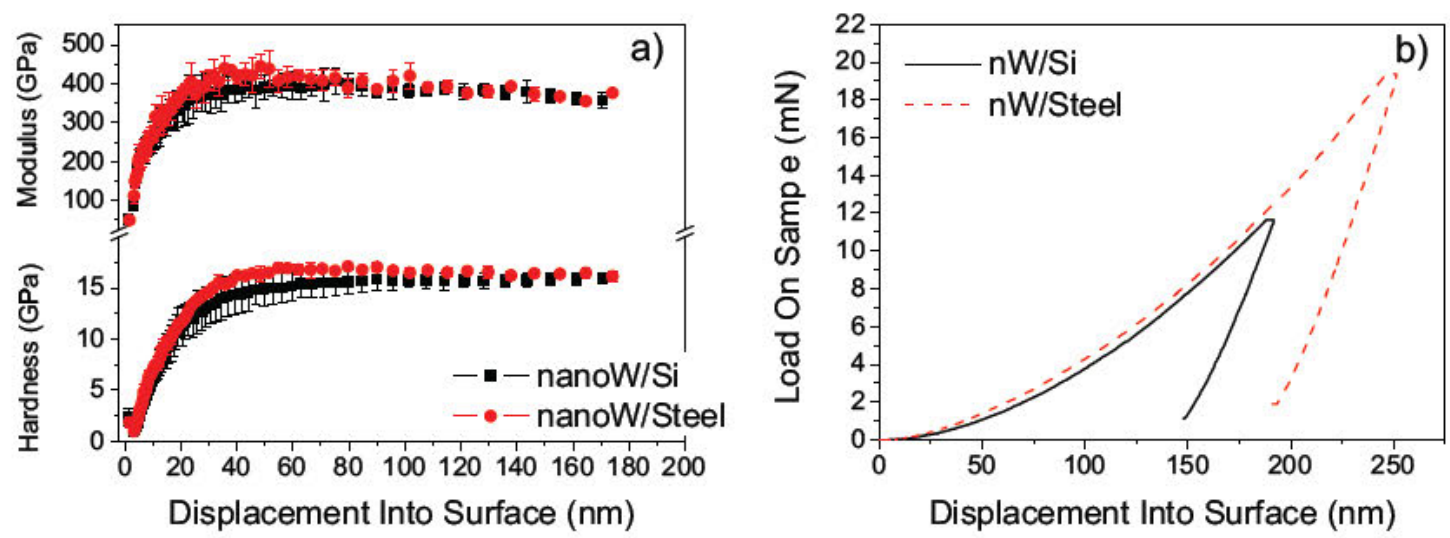

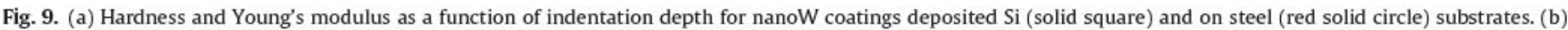

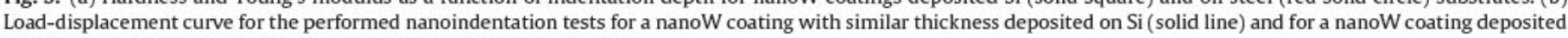
on steel (red dashed line) substrates (sample XI and V, respectively). 
total residual stress, Fig. 6(a)-(c), and low micro-strain, Fig. 7(b), thus, coating microstructure is similar to that for the coatings sputtered on (100) Si. The Hardness, Young's modulus and loaddisplacement curves for the sample deposited on steel are depicted in Fig. 9(a) and (b), respectively, as a function of indentation depth. The hardness and Young's modulus values are quite similar to those for the coatings deposited on (100) Si being $14.9 \pm 0.3 \mathrm{GPa}$ and $338 \pm 16 \mathrm{GPa}$, respectively. On these bases, we can conclude that the morphological, microstructural and mechanical properties for coatings deposited on commercial steel are comparable to those for coatings sputtered on ( 100$) \mathrm{Si}$.

\section{Conclusions}

The influence of sputtering power on deposition of nanow coating to the substrate has been investigated. Results evidence that non-delaminated coatings are achieved for sputtering power $\leq 50 \mathrm{~W}$.

Pure $\alpha$-W coatings were satisfactorily grown by DC-magnetron sputtering in wide range of thickness from $30 \mathrm{~nm}$ up to $4.0 \mu \mathrm{m}$ keeping the microstructure and morphology. For coatings deposited at a sputtering power of $50 \mathrm{~W}$, the dependence of the coating properties (morphology, microstructure, stress/strain state and mechanical response) on sample thickness has been investigated for films with thickness ranging from $250 \mathrm{~nm}$ up to $4.0 \mu \mathrm{m}$. No thickness dependence of the coatings properties was observed in the studied range. All investigated coatings consist of nanocolumns with an inverted pyramidal shape that grows perpendicular to the substrate surface. The average column diameter increases with the sample thickness. Microstructural studies illustrate that all the samples are polycrystalline and pure $\alpha-\mathrm{W}$ phase, having a strong $\{110\}$ texture. Coatings exhibit a compressive state with a total residual stress ranging from -2 up to $-5 \mathrm{GPa}$ and low micro-strain.

Nanoindentation tests evidenced that the coating hardness is as high as $\sim 15 \mathrm{GPa}$, a factor of 3.8 higher than that reported for coarse-grain W and the Young's modulus is lower $351 \pm 11 \mathrm{GPa}$ versus $410 \mathrm{GPa}$ reported for coarse grained $\mathrm{W}$, which may indicate that nanostructurization induce embrittlement. Further research is needed in order to clearly asses this point.

Finally, the morphology, microstructure, micro-strain and mechanical properties for samples deposited on (100) Si and on steel with similar thickness are pretty similar.

A deeper analysis on stress state and brittle-ductile transition for better understanding of the mechanical response are planned for the next work.

All presented results and the fact that coatings can be manufactured on steel substrates, suggest that the nanoW could be a good candidate for industrial applications.

\section{Acknowledgments}

The authors thanks to F. Munnik from HZDR for PIXE and RBS measurements and to J. Sousa from Nanotec Electrónica S.L. for the AFM images and. Research by N. Gordillo was supported by a Program for International Talent Recruitment (PICATA) postdoctoral fellowship of the Moncloa Campus of International Excellence (UCM-UPM). E. Tejado was supported by the CSIC (JAE-Predoc program) co-financed by FSE supports. I. Fernandez-Martinez was supported by a JdC program. This research was supported by Comunidad de Madrid (S-S2009/MAT-1585), EFDA-ITER (WP12-MAT-W ALLOY), Ministry of Economy and Competitiveness of Spain Government (ENE2012-39787-C06-0XX and MAT2012-38541-C02-01 and MAT2012-38541-C02-02).

\section{References}

[1] G. Bai, S. Wittenbrock, V. Ochoa, R. Villasol, C. Ciang, T. Marieb, D. Gardner, C. Mu, D. Fraser, M. Bohr, Mater. Res. Soc. Symp. Proc. 403 (1996) 501.

[2] J.S. Reid, E. Kolawa, R. Ruiz, M.A. Nicolet, Thin Solid Films 236 (1993) 319.

[3] R. Restani, A. Walchli, 12th European Workshop on Modern Developments in Microbeam Analysis, IOP Conference Series-Materials Science and Engineering 2012, p. 012022, 32

[4] K. Cho, L. Kecskes, R. Dowding, B. Schuster, Q. Wei, R.Z. Valiev, Proceedings of the 25th Army Science Conference, Orlando, FL, 27, 2006, ARL-RP-180.

[5] V. Barabash, G. Federici, R. Matear, A.R. Raffray, ITER Home Teams, Phys. Scr. 81 (1999) 74 .

[6] M. Kaufmann, R. Neu, Fusion Eng. Des. 82 (2007) 521

[7] R. Matera, G. Federici, The ITER Joint Central Team, J. Nucl. Mater. 233-237 (1996) 17.

[8] Y. Ueda, Plasma Fusion Res. 5 (2010) S1009.

[9] T.J. Renk, P. Provencio, T.J. Tanaka, J. Blanchard, C.J. Martin, T.R. Knowles, Fusion Sci. Technol. 61 (2012) 57.

[10] G.L. Kerr, D.C. Cox, V. Stolojan, S.R. Silva, J. Phys. Conf. Ser. 126 (2008) 012073.

[11] R. Vladoiu, V. Ciupina, C. Lungu, V. Bursikova, G. Musa, J. Optoelectron. Adv. Mater. 8 (2006) 71

[12] A. Bailini, F. Di Fonzo, M. Fusi, C.S. Casari, A. Li Bassi, V. Russo, A. Baserga, C.E Bottani, Appl. Surf. Sci. 253 (2007) 8130.

[13] J. Bloch, M. Heiblum, Y. Komem, Appl. Phys. Lett. 46 (1985) 1092.

[14] Q. Wei, H.T. Zhang, B.E. Schuster, K.T. Ramesh, R.Z. Valiev, L.J. Kecskes, R.J. Dowding, L. Magness, K. Cho, Acta Mater. 54 (2006) 4079.

[15] R.C. Sun, T.C. Tisone, D. Cruzan, J. Appl. Phys. 46 (1975) 112.

[16] R. Koch, J. Phys. Condens. Matter 6 (1994) 9519.

[17] T.J. Vink, W. Walrave, J.L.C. Daams, A.G. Dirks, M.A.J. Somers, K.J.A. van der Aker J. Appl. Phys. 74 (1993) 988.

[18] H. Maier, J. Luthin, M. Balden, J. Linke, F. Koch, H. Bolt, Surf. Coat. Technol. $142-144(2001) 733$

[19] A. Bensaoula, J.C. Wolfe, A. Ignatiev, F.O. Fong, T.S. Leung, J. Vac. Sci. Technol., A 2 (1984) 389.

[20] D.L. Windt, J. Vac. Sci. Technol., B 17 (1999) 1385.

[21] T. Karabacak, C.R. Picu, J.J. Senkevich, J. Appl. Phys. 96 (2004) 5740.

[22] T. Karabacak, Jay. J. Senkevich, Gwo-Ching Wang, Toh-Ming Lu, J. Vac. Sci. Technol., A 23 (2005) 986.

[23] J.A. Thornton, J.A. Hoffman, J. Vac. Sci. Technol. 40 (1977) 164.

[24] 〈www.nano4energy.eu〉.

[25] I. Horcas, R. Fernández, J.M. Rodríguez, J. Colchero, J. Gómez-Herrero, M. Baro, Rev. Sci. Instrum. 78 (2007) 013705.

[26] 〈www.hzdr.de〉.

[27] W.C. Oliver, G.M. Pharr, J. Mater. Res. 7 (1992) 1654.

[28] J.A. Thornton, J. Vac. Sci. Technol. 12 (1975) 830.

[29] G. Ackland, Science 327 (2010) 1587

[30] K.R. Khedir, G.K. Kannarpady, H. Ishihara, J. Woo, C. Ryerson, A.S. Biris, Phys. Lett. A 374 (2010) 4430

[31] Erik Lassner, Wolf-Dieter Schubert, “Tungsten” Cap, first ed., Springer, United States of America, 1999, 0-306-15053-4.

[32] B. Girault, D. Eyidi, T. Goudeau, Sauvage, E. Guerin, Bourhis Le, P.-O. Renault, J. Appl. Phys. 133 (2013) 174310

[33] M.J. O’Keefe, J.T. Grant, J. Appl. Phys. 79 (1996) 9134.

[34] Y.G. Shen, Y.W. Mai, J. Mater. Sci. 36 (2001) 93.

[35] R. Gonzalez-Arrabal, A. Camón, M. Parra-Borderías, L. Fabrega, J. Anguita, J. Sesé, F. Briones, J. Low Temp. Phys. 151 (2008) 239.

[36] N. Gordillo, R. Gonzalez-Arrabal, M.S. Martin-Gonzalez, J. Olivares, A. Rivera, F. Briones, F. Agullo-Lopez, D.O. Boerma, J. Cryst. Growth (2008) 4362.

[37] H.E. Swanson, E. Tatge, Natl. Bur. Stand. (U.S.) Circ. 539 (I) (1953) 28.

[38] I.C. Noyan, C.C. Goldsmith, JCPDS-ICDD, 1997.

[39] L.M. Kaatz, A.E. Lita, D. Balzar, Proceeding in Conference: Utah Space Grant Consortium, 2004.

[40] P.K. Predecki, I.C. Noyan, JCPDS-ICDD T Ely, 1999

[41] ISTE Ltd, 2007 British Library, X-ray Diffraction by Polycrystalline Materials, Chapter 6, René Guinebretière. ISBN 13: 978-1-905209-21-7.

[42] S. Eroglu, H. Ekren, T. Baykara, Scr. Mater. 38 (1997) 131.

[43] H.L. Sun, Z.X. Song, D.G. Guo, F. Ma, K.W. Xu, J. Mater. Sci. Technol. 26 (2010) 87 